\title{
Sex Differences in Association Between Anti-Hypertensive Medications and Risk of COVID-19 in Middle-Aged and Older Adults
}

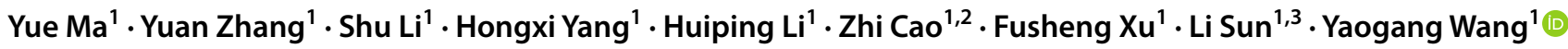

Accepted: 8 July 2021 / Published online: 18 August 2021

(c) The Author(s), under exclusive licence to Springer Nature Switzerland AG 2021

\begin{abstract}
Background There is ongoing debate about the associations between drug therapies targeting the renin-angiotensin-aldosterone system (RAAS) and adverse outcomes in coronavirus disease 2019 (COVID-19).

Objective This study aims to examine the associations between using medications for the cardiovascular system and the risks associated with COVID-19 in middle-aged and older adults.

Methods A total of 77,221 participants (aged 50-86 years) from UK Biobank were tested for SARS-CoV-2 RNA. The medications included angiotensin-converting enzyme inhibitors (ACEI), angiotensin-receptor blockers (ARB), $\beta$-blockers, calcium channel blockers (CCB), statins, and aspirin. COVID-19 outcomes comprised a positive test result and severity of COVID-19 (defined as mild, hospitalization or death). We evaluated the risk among total participants and for sub-groups based on sex. Propensity score matching was performed 1:1 and logistic regression models were used.

Results Among the middle- and older aged participants, no significant associations between any class of medications and the likelihood of COVID-19 infection were observed. ACEI were associated with a higher mortality risk from COVID-19 (odds ratio $[\mathrm{OR}] 1.15,95 \%$ confidence interval [CI] 1.01-1.32) and $\mathrm{CCB}$ were associated with a lower hospitalization risk for COVID-19 (OR 0.87, 95\% CI 0.79-0.96) among the male patients with COVID-19, while a lower mortality risk from COVID-19 (OR 0.67, 95\% CI 0.47-0.96) was observed with ARB among the female patients with COVID-19.

Conclusions The study suggested sex differences in the risk of death from COVID-19 with the use of ACEI and ARB among middle-aged and older adults. Sex differences in the risk of hospitalization for COVID-19 with the use of CCB was observed as well. It is of clinical importance that clinicians adopt different CVD treatment approaches for female and male patients with COVID-19.
\end{abstract}

Yaogang Wang

YaogangWANG@tmu.edu.cn

1 School of Public Health, Tianjin Medical University, Tianjin 300070, China

2 Department of Epidemiology and Health Statistics, School of Public Health, Zhejiang University, Hangzhou, China

3 School of Nursing, Tianjin Medical University, Tianjin, China

\section{Key Points}

It is important to determine sex differences in the association between using renin-angiotensin-aldosterone system (RAAS) inhibitors and the risks of COVID-19.

Sex differences in the association between RAAS inhibitors and risks of COVID-19 may be stronger at older ages.

There was a potential mortality risk for male patients with COVID-19 in using angiotensin-converting enzyme inhibitors and a protective effect of using calcium channel blockers. For female patients with COVID-19, using angiotensin-receptor blockers was associated with a lower risk of COVID-19 mortality. 


\section{Introduction}

In 2020, the rapid spreading of coronavirus disease 2019 (COVID-19) has forced the World Health Organization (WHO) to declare COVID-19 as a global pandemic. According to the WHO COVID-19 dashboard, more than 173 million people were confirmed to have contracted COVID-19, including 3 million deaths [1].

As the COVID-19 pandemic has spread, there has been a growing recognition that pre-existing cardiovascular diseases (CVD) and risk factors in middle-aged and older adults can increase the severity of COVID-19, leading to the aggravation and decompensation of chronic underlying cardiac pathologies as well as acute onset of new cardiac complications [2]. This phenomenon suggests that severe acute respiratory syndrome coronavirus 2 (SARS-CoV-2, the etiological agent of COVID-19) infection may result in activation of related signaling pathways in the cardiovascular system [3].

S-proteins located on the surface of SARS-CoV-2 can bind to the cell receptor, angiotensin-converting enzyme (ACE) 2 [4], which is part of the renin-angiotensin-aldosterone system (RAAS) and its neurohormonal pathways [5]. As a result, a heated argument is currently underway about the association between medical therapy for CVD and COVID-19 severity, and thereby the possibility of these drugs increasing the severity of infection [6]. The most controversial argument is that treatment with RAAS inhibitors can increase tissue expression of ACE2 and its presentation at the cell surface in certain clinical states [7].

The main reason for the heated discussion is the inconsistent results in previous studies. One study conducted in the United States suggested that anti-hypertensive medications were associated with no substantial increase in risk for COVID-19 patients [8]. Another study found that hospitalized patients with COVID-19 using ACE inhibitors (ACEI) or angiotensin II receptor blockers (ARB) were associated with lower risk of all-cause mortality compared with nonusers [9]. Further fueling these concerns is the observation that using ACEI or ARB might increase the risk of COVID19 after exposure to SARS-CoV-2 [10]. Except for the common anti-hypertensive medications, studies found a higher risk of death was associated with atorvastatin [11], and safety issues in using aspirin related to the risk of bleeding and serious liver damage in patients with COVID-19 [12].

However, these studies have recognized that gender might contribute to the inconsistent results. Gender and age play important roles in drug efficacy and COVID-19 outcomes. A recent study showed that treatment with ACEI or ARB could reduce the mortality rate from hyperkalemia and acute kidney injury in women, but not men [13]. The expression of ACE2 and regulatory mechanisms of the cardiovascular system varied by sex $[14,15]$. Sex differences in COVID19 are becoming more apparent as mounting data indicate that males seem to be disproportionately at risk of a severe COVID-19 outcome due to preexisting CVD and COVID19-related cardiovascular injury [16].

Owing to the growing sex gap in patients with COVID19 [17], exploring the relationship between cardiovascular medications and COVID-19 outcomes in different sexes is very important for clinical treatment. We sought to estimate the association between using cardiovascular medications and the risk of testing positive, hospitalization, and death from COVID-19 among male and female participants from UK Biobank.

\section{Methods}

\subsection{Study Population}

The study included 77,221 participants who had been tested for SARS-CoV-2 RNA from March 16, 2020 to May 19, 2021 from UK Biobank. UK Biobank includes over 500,000 middle- and older aged adults who have undergone detailed medical and cognitive phenotypic assessment. All the UK Biobank participants gave written informed consent before data collection. UK Biobank has full ethical approval from the NHS National Research Ethics Service (http://www. ukbiobank.ac.uk/ethics/). This work was conducted under UK Biobank application number 45676.

\subsection{Medication and History Assessment}

Medical history was extracted from UK Biobank that had been entered into the medical history or problem list sections of the medical record or since encounter diagnoses at the time of enrollment (2006-2010) and follow-up period. We selected the six classes of cardiovascular drugs that require lifelong prescriptions, including ACEI, ARB, $\beta$-blockers, calcium channel blockers (CCB), statins, and aspirin.

The history of disease was defined as a self-reported, physician-diagnosed, primary care, or hospital record before May 19, 2021. We characterized the participants as having or not having a history of chronic obstructive pulmonary disease (according to the International Classification of Diseases [ICD] 10th edition [ICD-10] codes J41, J42, J43, J44; ICD 9th edition [ICD-9] codes 491, 492, 496), chronic kidney disease (ICD-10: N18; ICD-9: 5859; self-report code: 1192), diabetes (ICD-10: E10-E14.9), hypertension (ICD10: I10-I16, H35.03, I67.4, O10.1-O10.4, O10.9), coronary artery disease (ICD-10: I20-I25), heart failure (ICD-10: I50, I11.0, I13.0), stroke (ICD-10: F01, G45, G46, I60-64, I690, I691, I692, I694, I698, I699), pulmonary embolism 
(ICD-10: I26; ICD-9: 415), and asthma (ICD-10: J44-46; ICD-9: 493).

\subsection{Outcomes of COVID-19}

The primary outcome was the risk of positive SARS-CoV-2 tests, and the secondary outcome was the risk of severity in COVID-19-positive patients. We assessed the primary and secondary outcomes in total patients, and in male and female participants, separately. Participants were deemed to be positive for COVID-19 if any test was positive for SARS-CoV-2 RNA and negative if all tests were negative. For the patients with a positive COVID-19 test result, we classified them as mild, hospitalized, and dead. Patients were identified as mild cases of COVID-19 if they were without any record for hospitalization or death. COVID-19-specific death was defined as cause of death due to ICD-10 U07.1 using death registry data linked to UK Biobank.

\subsection{Statistical Analysis}

The characteristics of the study population were compared by sex using t tests for continuous variables and chi-squared tests for categorical variables. The continuous variables were described as mean $\pm \mathrm{SD}$. The categorical variables were described as sums with percentages. If data was missing for a covariate, we used multiple imputations based on five replications and utilized a chained-equation method to account for the missing data. We conducted a complete case analysis to show whether the imputation process introduced bias to our outcome. A two-tailed $p$-value $<0.05$ was considered statistically significant.

To reduce confounding effects of potential risk factors on outcomes, 1:1 propensity score matching (PSM) was applied to match users of medications and nonusers into treated and control groups among total participants. Total participants were propensity-score matched for age, sex, race, body-mass index, smoking history, systolic and diastolic blood pressure, history of chronic obstructive pulmonary disease, chronic kidney disease, diabetes mellitus, hypertension, coronary heart disease, heart failure, stroke, pulmonary embolism, asthma and other classes of medication. Age was recalculated at the time of the test results for COVID-19. The covariates of PSM for male and female participants are the same as the total participants except for gender. Propensity scores were generated using a multivariable logistic regression model. PSM was implemented with a nearest-neighbor strategy, width of caliper was 0.02 . No replacement was allowed, and patients were matched only once. Standardized mean differences (SMD) before and after matching are shown in Supplementary Appendix (see electronic supplementary material [ESM]). The balances of matched covariates were evaluated with SMD, and $<10 \%$ differences were considered matched sufficiently [18]. We also conducted stratified analyses by sex (men and women). The interaction test between sex and each class of medication was performed by using the likelihood ratio test comparing models with and without a cross-product term. The association between six classes of medication and risk of a COVID-19-positive result and severity of COVID-19 was examined by logistic regression. We conducted sensitivity analyses using multivariate adjusted logistic regression rather than propensityscore matching. Effect of medicine class was considered significant at a $p$-value $<0.05$. Analyses were performed with R statistical software, version 4.0.3 (with the libraries MatchIt and Survival).

\section{Results}

\subsection{Characteristics of the Participants}

A total of 77,221 participants aged from 50 to 86 years who had COVID-19 test results in UK Biobank were included. These participants were classified into two subgroups by sex; 35,892 (46.5\%) were men and 41,329 (53.5\%) were women (Fig. 1). Among the male population, 4819 (13.4\%) tested positive, including 2030 (42.1\%) mild cases (tested positive without hospitalization or death), 2526 (52.4\%) who were hospitalized, and 263 (5.5\%) deaths. Among the female population, $5676(13.7 \%)$ tested positive, including 2612 (46.0\%) mild cases, 2875 (50.7\%) hospitalized cases, and $189(3.3 \%)$ deaths.

Men had a higher prevalence of severe COVID-19 compared with women $(p<0.001$, Table 1$)$. In addition, more men used the six classes of medication than women $(p<0.001$, Table 1), which underscores the importance of sex.

The complete case analysis showed that the imputation process introduced no bias to our results ( $p>0.05$, Supplementary Appendix Table S19, see ESM). After 1:1 PSM, medication users and matched nonusers were selected for analysis. In these two groups, the characteristics of the population were well balanced (all SMD $<0.1$ and $p>0.05$ ). Distribution of propensity scores and the histogram of propensity scores before and after matching in the control and medication-treated groups among total, male, and female participants are shown in Figs S1-S6 (see ESM). 


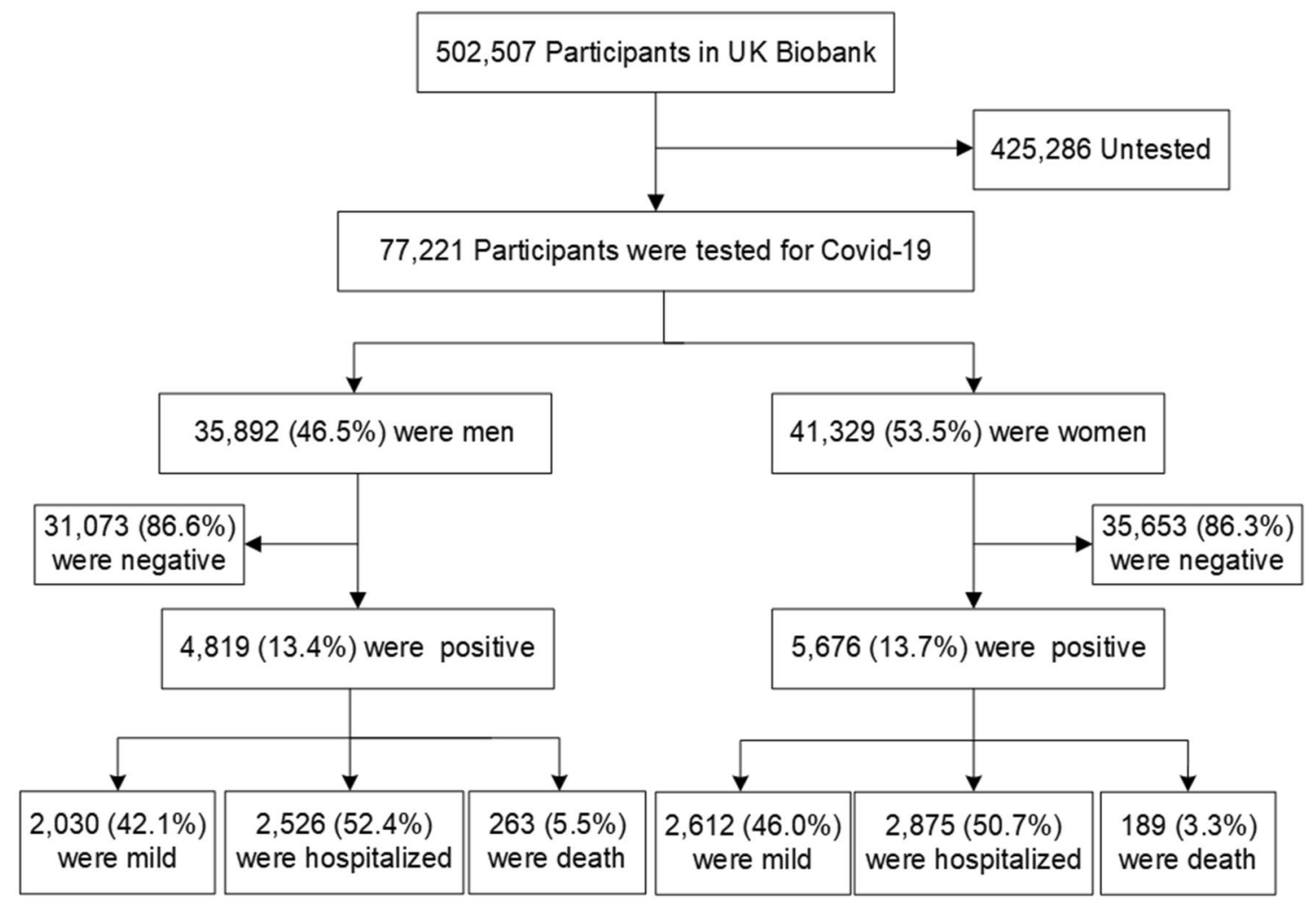

Fig. 1 Flowchart of UK Biobank participants selected for analyses

\subsection{Anti-Hypertensive Medication and COVID-19 in Total Participants}

A total of 77,221 participants were tested for COVID-19, including 10,495 (13.6\%) who tested positive for COVID-19 (Table 1). Among the 10,495 (13.6\%) COVID-19-positive patients, $4642(6.0 \%)$ were mild cases, $5401(7.0 \%)$ were hospitalized, and there were $452(0.6 \%)$ deaths.

The results of PSM quality among total participants are shown in Tables S1-S6 (see ESM). As shown in Fig. 2, the odds ratio (OR) of death from COVID-19 associated with ACEI and ARB classes of therapy in the PSM cohort was 1.00 (95\% confidence interval [CI] 0.89-1.13) and 0.85 (95\% CI 0.70-1.02), respectively. No classes of medication were associated with the risk of SARS-CoV-2 infection $(p>0.05$, Fig. 2). Similar results were also observed in the risk of COVID-19 severity analysis; neither ACEI nor ARB medication was associated with the risk of COVID19 severity in total participants (Fig. 2). $\beta$-blockers, CCB, statins, and aspirin are not associated with the risk of being COVID-19-positive or of COVID-19 severity in total participants ( $p>0.05$, Fig. 2).

We also tested the interaction between sex and use of medication on the risk of COVID-19 outcomes. We found significant interactions between use of ACEI $\left(P_{\text {interaction }}=\right.$ $0.031)$, ARB $\left(P_{\text {interaction }}=0.034\right)$, and sex on the risk of death from COVID-19. In addition, the use of CCB showed a significant interaction with sex on the risk of hospitalization for COVID-19 ( $P_{\text {interaction }}=0.027$, Fig. 2$)$. Sensitivity analyses with the use of multivariate logistic regression showed similar conclusions among the total participants (Table S20, see ESM).

\subsection{Anti-Hypertensive Medication and COVID-19 in Men and Women}

As shown in Fig. 3, using one of the six classes of medication is not associated with the risk of testing positive for SARS-CoV-2 in men and women after PSM (1:1). The results of PSM quality for men are shown in Tables S7-S12, the results for women are shown in Tables S13-S18.

Among male participants, the OR for COVID-19 death associated with ACEI was 1.15 (95\% CI 1.01-1.32; $p=0.040)$, compared with an OR of 0.97 (95\% CI $0.79-1.19)$ for female participants. ACEI had a 1.15 -fold higher risk of COVID-19 death among male participants compared with nonusers, whereas ACEI medication was 
Table 1 Clinical characteristics of the study population of participants tested for SARS-CoV-2

\begin{tabular}{|c|c|c|c|c|}
\hline Characteristic & $\begin{array}{l}\text { Total } \\
(n=77,221)\end{array}$ & $\begin{array}{l}\text { Men } \\
(n=35,892)\end{array}$ & $\begin{array}{l}\text { Women } \\
(n=41,329)\end{array}$ & $p$-Value ${ }^{\mathrm{a}}$ \\
\hline Age & $68.58 \pm 8.28$ & $69.06 \pm 8.25$ & $68.16 \pm 8.29$ & $<0.001$ \\
\hline Race, $n(\%)$ & & & & $<0.001$ \\
\hline White & $72,225(93.5)$ & $33,609(93.6)$ & $38,616(93.4)$ & \\
\hline Asian & $2120(2.7)$ & $1127(3.1)$ & $993(2.4)$ & \\
\hline Black & $1559(2.0)$ & $626(1.7)$ & $933(2.3)$ & \\
\hline Other & $1317(1.7)$ & $530(1.5)$ & $787(1.9)$ & \\
\hline Smoking status & & & & $<0.001$ \\
\hline Never & $40,518(52.5)$ & $16,742(46.6)$ & $23,776(57.5)$ & \\
\hline Previous & $28,640(37.1)$ & $14,920(41.6)$ & $13,720(33.2)$ & \\
\hline Current & 8063 (10.4) & $4230(11.8)$ & $3833(9.3)$ & \\
\hline BMI & $27.89 \pm 4.97$ & $28.26 \pm 4.39$ & $27.56 \pm 5.40$ & $<0.001$ \\
\hline \multicolumn{5}{|l|}{ Comorbidities, n (\%) } \\
\hline COPD & $5662(7.3)$ & $3038(8.5)$ & $2624(6.3)$ & $<0.001$ \\
\hline Chronic kidney disease & $5879(7.6)$ & $3048(8.5)$ & $2831(6.8)$ & $<0.001$ \\
\hline Diabetes mellitus & $10,045(13.0)$ & $5929(16.5)$ & $4116(10.0)$ & $<0.001$ \\
\hline Hypertension & $33,333(43.2)$ & $17,520(48.8)$ & $15,813(38.3)$ & $<0.001$ \\
\hline Coronary heart disease & $12,971(16.8)$ & $8195(22.8)$ & $4776(11.6)$ & $<0.001$ \\
\hline Heart failure & $4549(5.9)$ & $2847(7.9)$ & $1702(4.1)$ & $<0.001$ \\
\hline Stroke & $4718(6.1)$ & $2730(7.6)$ & $1988(4.8)$ & $<0.001$ \\
\hline Pulmonary embolism & $1960(2.5)$ & $1051(2.9)$ & $909(2.2)$ & $<0.001$ \\
\hline Asthma & 9739 (12.6) & 3917 (10.9) & $5822(14.1)$ & $<0.001$ \\
\hline TDI & $-1.17 \pm 3.14$ & $-1.16 \pm 3.19$ & $-1.17 \pm 3.10$ & 0.671 \\
\hline \multicolumn{5}{|l|}{ Medications, n (\%) } \\
\hline ACEI & 9187 (11.9) & $5738(16.0)$ & $3449(8.3)$ & $<0.001$ \\
\hline ARB & $3354(4.3)$ & $1779(5.0)$ & $1575(3.8)$ & $<0.001$ \\
\hline$\beta$-blocker & $6360(8.2)$ & $3731(10.4)$ & $2629(6.4)$ & $<0.001$ \\
\hline $\mathrm{CCB}$ & $6158(8.0)$ & $3732(10.4)$ & $2426(5.9)$ & $<0.001$ \\
\hline Statin & $15,044(19.5)$ & $9276(25.8)$ & $5768(14.0)$ & $<0.001$ \\
\hline Aspirin & $12,471(16.1)$ & 7753 (21.6) & 4718 (11.4) & $<0.001$ \\
\hline Diastolic BP & $82.17 \pm 10.22$ & $83.91 \pm 10.11$ & $80.66 \pm 10.08$ & $<0.001$ \\
\hline Systolic BP & $138.27 \pm 18.71$ & $141.41 \pm 17.65$ & $135.55 \pm 19.18$ & $<0.001$ \\
\hline COVID-19 status & & & & $<0.001$ \\
\hline Negative & $66,726(86.4)$ & $31,073(86.6)$ & $35,653(86.2)$ & \\
\hline Positive & $10,495(13.6)$ & $4819(13.4)$ & $5676(13.7)$ & \\
\hline Mild COVID-19 cases & $4642(6.0)$ & $2030(5.6)$ & $2612(6.3)$ & \\
\hline Hospitalized for COVID-19 & $5401(7.0)$ & $2526(7.0)$ & $2875(6.9)$ & \\
\hline Death from COVID-19 & $452(0.6)$ & $263(0.7)$ & $189(0.4)$ & \\
\hline
\end{tabular}

Note: Mild COVID-19 cases comprise the participants that tested positive for COVID-19 without hospitalization or death

$A C E I$ angiotensin converting enzyme inhibitor, $A R B$ angiotensin receptor blocker, $B M I$ body mass index, $B P$ blood pressure, $C C B$ calcium channel blocker, COPD chronic obstructive pulmonary disease, COVID-19 coronavirus disease 2019, TDI Townsend deprivation index

${ }^{\text {a }}$ Comparison between men and women groups

not associated with the risks of COVID-19 among female participants.

Interestingly, the OR for COVID-19 death associated with ARB was 0.67 (95\% CI 0.47-0.96; $p=0.028$ ) among female participants, compared with an OR of 1.38 (95\% CI 0.87-1.78; $p=0.062$ ) for the male population. ARB decreased risk of death from COVID-19 by $33 \%$ in women compared with nonusers, whereas ARB medication is not associated with the risk of death in men. From the above, ACEI medication is a risk factor for COVID-19 death in men, while ARB is a protective factor for death from COVID-19 in women. 


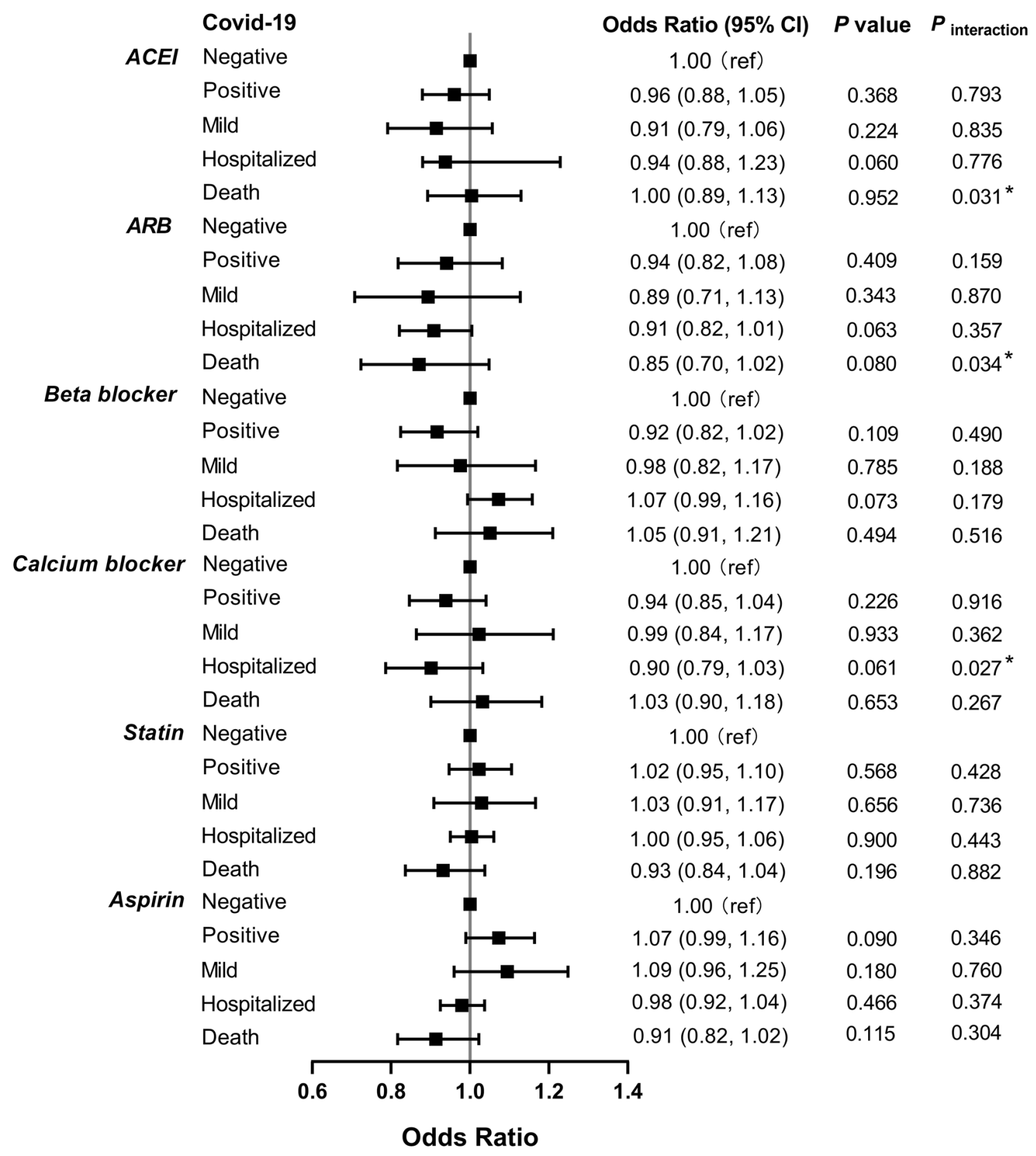

Fig. 2 Association between medications and the risk of COVID-19 after propensity score matching in total participants. ACEI angiotensin converting enzyme inhibitor, $A R B$ angiotensin receptor blocker,

What is more, the OR of hospitalization for COVID-19 associated with CCB in men was 0.87 (95\% CI 0.79-0.96; $p=0.005$ ), compared with an OR of 1.03 (95\% CI 0.86-1.15; $p=0.529$ ) for women. This suggests that CCB could decrease the risk of hospitalization for COVID-19 in men by $13 \%$, while CCB is not associated with the risk of hospitalization for COVID-19 in women. $\beta$-blockers, statins, and aspirin were not associated with COVID-19 outcomes in both females and males, similar to the total participants. Sensitivity analyses
$P_{\text {interaction }}, p$-value for interaction between the medication and sex on the risk of COVID-19

with the use of multivariate logistic regression showed similar conclusions among the male and female participants (Table S20, see ESM). 


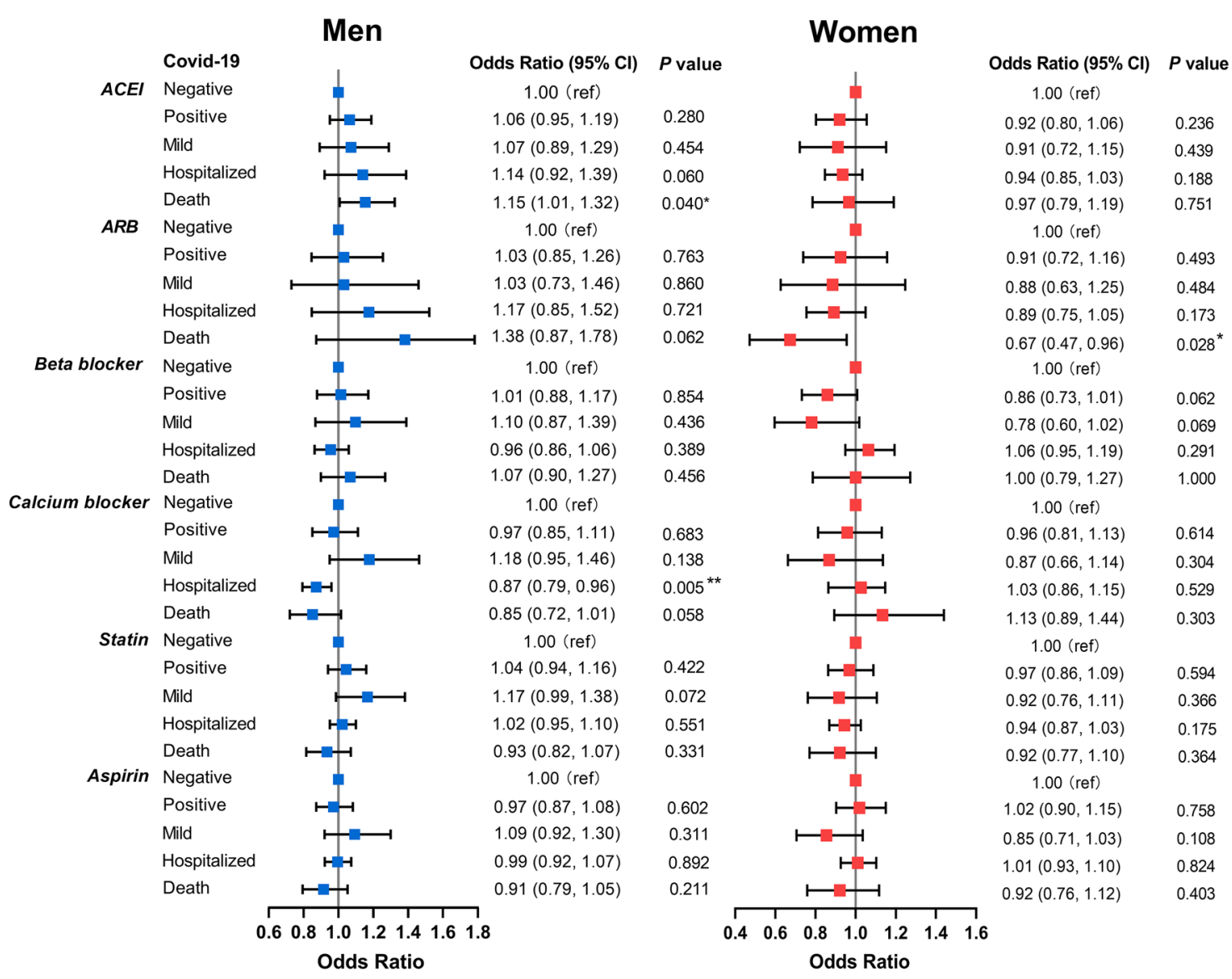

Fig. 3 Association between medications and the risk of COVID-19 after propensity score matching in men and women. The blue square is the odds ratio for the risk of COVID-19 outcomes in men. The red square is the odds ratio for the risk of COVID-19 outcomes in women. The effect of medicine classes was considered significant at a $p$-value of $<0.05$. $* p<0.05$ compared with COVID-19-negative participants; ${ }^{* *} p<0.01$ compared with COVID-19-negative participants

\section{Discussion}

This prospective cohort study suggested that treatment with medications (including ACEI, ARB, $\beta$-blockers, $\mathrm{CCB}$, statins and aspirin) acting on CVD was not associated with the risk of testing positive for COVID-19 in female, male, and overall participants. Among middleaged and older adults, the use of ACEI and ARB had different effects on the risk of death from COVID-19 and the use of CCB had different effects on the risk of hospitalization for COVID-19 in females and males. Among the male patients with COVID-19 in our cohort, using ACEI was associated with a higher risk of death for COVID-19 and using CCB was associated with a lower risk of hospitalization for COVID-19, while using ARB was associated with a lower risk of death from COVID-19 among the female COVID-19 patients in our cohort. This finding may contribute to the fierce debate since the discovery of SARSCoV-2 in the clinical community about whether patients with COVID-19 should continue to use the six classes of CVD medication.

Our data suggested that using ACEI increased the risk of death for COVID-19 in men and using ARB decreased the risk in women. These findings could be attributed to two aspects. First, and most importantly, because the expression of ACE2 on the X chromosome can be influenced by estrogen, the ACE/ACE2 ratio may be shifted towards the ACE2/ 
Angiotensin (Ang)-(1-7)/MAS receptor axis more in women than in men $[19,20]$. Some studies have described that the increased ACE2/Ang-(1-7)/MAS pathway activity in the female reproductive system can be an important mechanism to counteract the actions of the Ang II-AT1R pathway [21]. The activity of the ACE2/Ang-(1-7)/MAS pathway is beneficial for the prognosis of CVD in women (the mechanism is shown as Fig. 4) [22]. In contrast, the expression of ACE2 was found more in men than in women, which could cause higher incidence and fatality rate of COVID-19 in men [23].

We also found that CCB was associated with a lower risk of hospitalization for COVID-19 in males. The potential mechanism may be that inhibited calcium entry into cells can affect vital steps in the life cycle of the viruses [24, 25] (Fig. 4) and the modulation of calcium channel responsiveness is sex-specific. Animal studies have proved that male papillary heart muscle is more sensitive to calcium than female due to the regulation of calcium channel expression and current by estrogen and testosterone [26, 27].

Sex differences in cardiovascular disease and COVID19 share mechanistic foundations, namely, the involvement of both the innate immune system and the canonical renin-angiotensin system. Immunological differences suggest that females mount a rapid and aggressive innate immune response, and the attenuated antiviral response in males may confer enhanced susceptibility to severe disease [28]. Although previous studies rarely reported sex differences in the use of RAAS inhibitors among patients with COVID-19, the sex differences have been established in terms of CVD onset, pathophysiology, manifestation, susceptibility, prevalence, treatment responses and outcomes in animal models and clinical populations [29]. Apart from that, many clinical studies have found that there was no difference in the prevalence of COVID-19 between females and males, but male patients had significantly higher risk of severe illness or death than female patients [30-32].

Furthermore, a cohort study using data from the Korean National Health Insurance Service including 1,374,381 residents found that there was a significantly lower risk of COVID-19 for participants using ARB and CCB medication [33]. Studies have shown the association between ARB and the lower risk of death for COVID-19, while ACEI was not associated with severity of COVID-19 [34, 35]. The effect of ACEI and ARB on ACE2 may be different [36]. A study

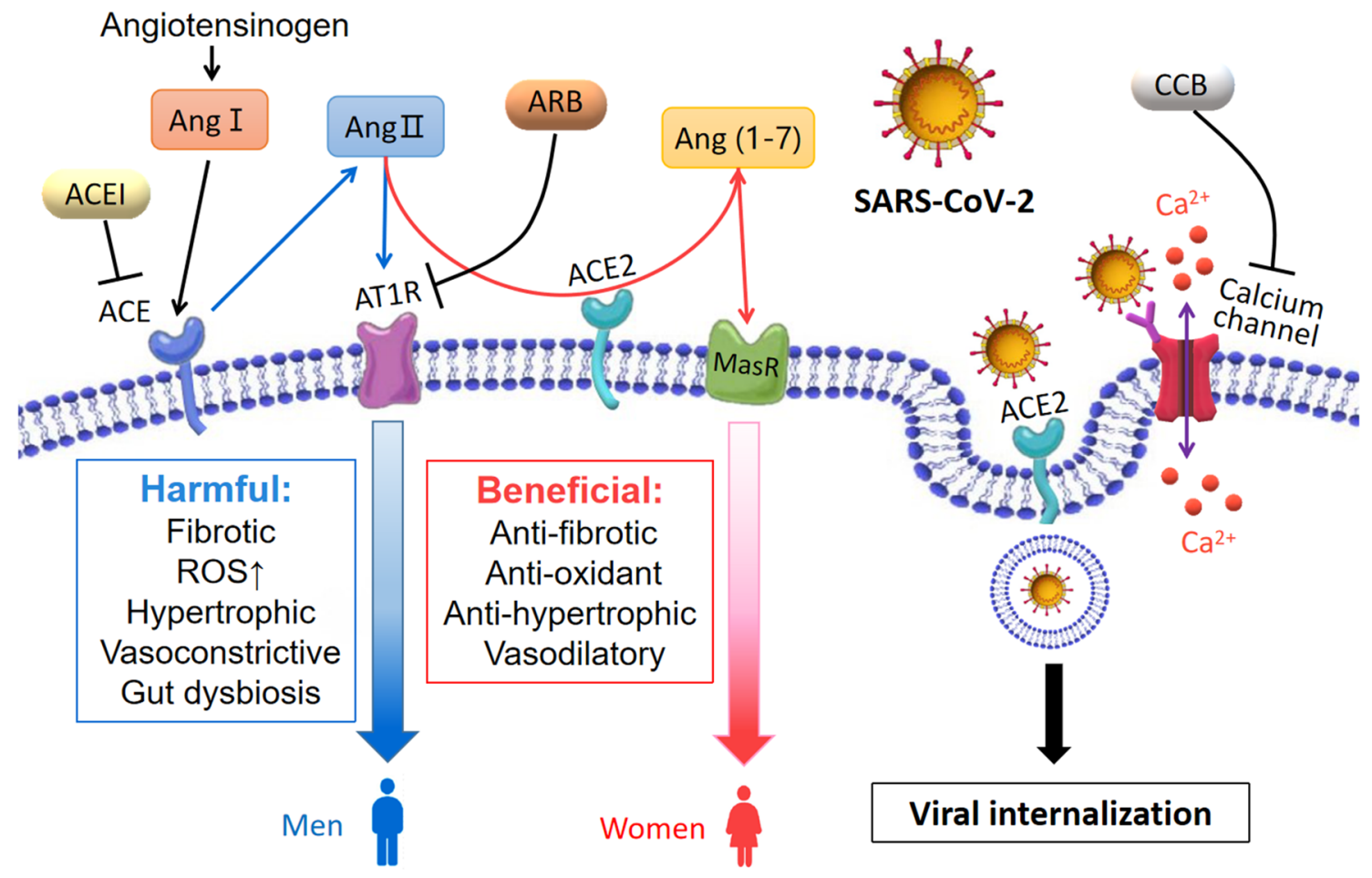

Fig. 4 Mechanisms for engagement of RAAS with SARS-CoV-2 in different sexes. Angiotensinogen is produced in the liver and hydrolyzed into angiotensin I (AngI). Angiotensin converting enzyme $(A C E)$ converts AngI into angiotensin II (AngII). ACE2 converts AngII into angiotensin 1-7 (Ang 1-7), which binds to Mas receptor (MasR) and favors tissue protection, mainly by hypotensive and antiinflammatory pathways among women. Conversely, ACE converts Ang I into Ang II that binds to angiotensin II type 1 receptor $(A T 1 R)$, favoring tissue injury among men. SARS-CoV-2 may interact with calcium channels to facilitate entry of $\mathrm{Ca}^{2+}$ and create a suitable environment for replication and survival. Calcium channel blockers $(C C B)$ inhibit the utilization of calcium channels by SARS-CoV-2 for survival. $A C E I$ angiotensin converting enzyme inhibitor, $A R B$ angiotensin receptor blocker, $R A A S$ renin-angiotensin-aldosterone system, $R O S$ reactive oxygen species, $S A R S-C o V-2$ severe acute respiratory syndrome coronavirus 2 
conducted in the National Health Insurance (NHI) program of Taiwan found that the use of ACEI is associated with an increased risk of lung cancer compared with the use of ARB. Patients using ARB have a significantly lower risk of lung cancer than non-ARB users [37]. In our study, $\beta$-blockers, statins, and aspirin were found to have no association with the risks of COVID-19, and these findings are in line with previous studies [5, 38, 39].

A population-based case-control study from the Lombardy region of Italy have identified no sex differences or effects of using ACEI/ARB on the risk of COVID-19 [40]. Another Italian observational multicenter survey found no significant interference between hypertension or anti-hypertensive therapy on COVID-19 lethality [41]. Differences in the research design and covariates or other methodological differences between our study and the two studies from Italy might contribute to the different findings. For example, in our study, the covariates include nine kinds of comorbidities and the case control includes four kinds of comorbidities.

The obvious advantage of our study compared with previous studies is the detailed and validated data in a well characterized cohort including types of cardiac medications and potential confounding risk factors for both females and males. Our findings might help to allay concerns that using the six classes of medications increases the risk of SARS-CoV-2 infection. Also, using ACEI, ARB and CCB was found to have a different effect on COVID-19 severity between females and males. However, due to the limitations of the data in UK Biobank, this data may not reflect changes in actual drug exposure during follow-up and we did not bring health care insurance access into the covariates of PSM.

\section{Conclusions}

This study suggests sex differences in the risk of COVID-19 severity with ACEI, ARB, and CCB medications. We found a potential mortality risk for male patients with COVID-19 using ACEI and a protective effect of using CCB on the risk of hospitalization for COVID-19, while a protective effect of using ARB on the mortality of female patients with COVID19 was identified. Thus, it is of clinical importance that clinicians adopt different CVD treatment approaches for female and male patients with COVID-19. Indeed, the potentially deleterious effects of routine anti-hypertensive medication treatment on COVID-19-related mortality demand further investigation; in particular, well designed and powered randomized controlled trials will be able to properly address this important issue.

Supplementary Information The online version contains supplementary material available at https://doi.org/10.1007/s40266-021-00886-y.
Acknowledgements The present analyses were conducted using the UK Biobank resource under Application 45676. We would like to thank all UK Biobank participants and staff, and all health-care workers involved in the diagnosis and treatment of COVID-19 patients. We acknowledge support to Y.G.W. The funding sources had no involvement in the study design, collection, analysis, or interpretation of data.

\section{Declarations}

Funding This study was supported by The National Natural Science Foundation of China (71910107004).

Conflicts of interest The authors declare no conflict of interest.

Ethics approval This article does not contain any studies with human participants or animals performed by the authors.

Data availability The authors can confirm that all relevant data are included in the article and/or its supplementary information files.

Authors' contributions YW directed the study. YW and YM designed the study and analyzed data. YM developed the first manuscript draft. YW, SL, HY and YZ edited the manuscript. All authors critically revised the manuscript, and all authors contributed to the final version.

\section{References}

1. WHO Coronavirus Disease (COVID-19) Dashboard. 2021. https:// covid19. who.int/. Accessed 9 Jun 2021.

2. Guo T, Fan Y, Chen M, Wu X, Zhang L, He T, et al. Cardiovascular implications of fatal outcomes of patients with Coronavirus disease 2019 (COVID-19). JAMA Cardiol. 2020;5(7):811-8.

3. Driggin E, Madhavan MV, Bikdeli B. Cardiovascular considerations for patients, health care workers, and health systems during the COVID-19 pandemic. J Am Coll Cardiol. 2020;75(18):2352-71.

4. Ziegler CGK, Allon SJ, Nyquist SK, Mbano IM, Miao VN, Tzouanas $\mathrm{CN}$, et al. SARS-CoV-2 receptor ACE2 is an interferon-stimulated gene in human airway epithelial cells and is detected in specific cell subsets across tissues. Cell. 2020;181(5):1016-35.

5. Vaduganathan M, Vardeny O, Michel T, McMurray JJV, Pfeffer MA, Solomon SD. Renin-Angiotensin-Aldosterone system inhibitors in patients with Covid-19. N Engl J Med. 2020;382(17):1653-9.

6. South AM, Tomlinson L, Edmonston D, Hiremath S, Sparks MA. Controversies of renin-angiotensin system inhibition during the COVID-19 pandemic. Nat Rev Nephrol. 2020;16(6):305-7.

7. Serfozo P, Wysocki J, Gulua G, Schulze A, Ye M, Liu P, et al. Ang II (Angiotensin II) conversion to angiotensin-(1-7) in the circulation Is POP (Prolyloligopeptidase)-dependent and ACE2 (Angiotensin-Converting Enzyme 2)-independent. Hypertension. 2020;75(1):173-82.

8. Reynolds HR, Adhikari S, Pulgarin C, Troxel AB, Iturrate E, Johnson $\mathrm{SB}$, et al. Renin-Angiotensin-Aldosterone system inhibitors and risk of Covid-19. N Engl J Med. 2020;382(25):2441-8.

9. Zhang P, Zhu L, Cai J, Lei F, Qin JJ, Xie J, et al. Association of inpatient use of Angiotensin-converting enzyme inhibitors and Angiotensin II receptor blockers with mortality among patients with hypertension hospitalized with COVID-19. Circ Res. 2020;126(12):1671-81.

10. Guo J, Huang Z, Lin L, Lv J. Coronavirus disease 2019 (COVID-19) and cardiovascular disease: a viewpoint on the potential influence 
of angiotensin-converting enzyme inhibitors/angiotensin receptor blockers on onset and severity of severe acute respiratory syndrome coronavirus 2 infection. J Am Heart Assoc. 2020;9(7):e016219.

11. Cariou B, Goronflot T, Rimbert A, Boullu S, Le May C, Moulin P, et al. Routine use of statins and increased COVID-19 related mortality in inpatients with type 2 diabetes: results from the CORONADO study. Diabetes Metab. 2021;47(2):101202.

12. Bianconi V, Violi F, Fallarino F, Pignatelli P, Sahebkar A, Pirro M.Is acetylsalicylic acid a safe and potentially useful choice for adult patients with COVID-19? Drugs. 2020;80(14):1383-96.

13. Dumanski SM, Ahmed SB. Sex, drugs, and adverse drug reactions: sex differences in laboratory monitoring outcomes after initiation of renin-angiotensin-aldosterone-system inhibition therapy. Circ Cardiovasc Qual Outcomes. 2020;13(9):e007045.

14. Lukassen S, Chua RL, Trefzer T, Kahn NC, Schneider MA, Muley T, et al. SARS-CoV-2 receptor ACE2 and TMPRSS2 are primarily expressed in bronchial transient secretory cells. EMBO J. 2020;39(10):e105114.

15. Domińska K. Involvement of ACE2/Ang-(1-7)/MAS1 axis in the regulation of ovarian function in mammals. Int $\mathbf{J}$ Mol Sci. 2020;21(13):4572.

16. Medzikovic L, Cunningham CM, Li M, Amjedi M, Hong J, Ruffenach G, et al. Sex differences underlying preexisting cardiovascular disease and cardiovascular injury in COVID-19. J Mol Cell Cardiol. 2020;148:25-33.

17. La Vignera S, Cannarella R, Condorelli RA, Torre F, Aversa A, Calogero AE. Sex-specific SARS-CoV-2 mortality: among hormonemodulated ACE2 expression, risk of venous thromboembolism and hypovitaminosis D. Int J Mol Sci. 2020;21(8):2948.

18. Austin PC. Optimal caliper widths for propensity-score matching when estimating differences in means and differences in proportions in observational studies. Pharm Stat. 2011;10(2):150-61.

19. Li Y, Zhou W, Yang L, You R. Physiological and pathological regulation of ACE2, the SARS-CoV-2 receptor. Pharmacol Res. 2020;157:104833.

20. Tukiainen T, Villani AC, Yen A, Rivas MA, Marshall JL, Satija R, et al. Landscape of $\mathrm{X}$ chromosome inactivation across human tissues. Nature. 2017;550(7675):244-8.

21. Bessa ASM, Jesus ÉF, Nunes ADC, Pontes CNR, Lacerda IS, Costa JM, et al. Stimulation of the ACE2/Ang-(1-7)/Mas axis in hypertensive pregnant rats attenuates cardiovascular dysfunction in adult male offspring. Hypertens Res. 2019;42(12):1883-93.

22. Santos RAS, Sampaio WO, Alzamora AC, Motta-Santos D, Alenina $\mathrm{N}$, Bader M, et al. The ACE2/Angiotensin-(1-7)/MAS axis of the Renin-Angiotensin system: focus on Angiotensin-(1-7). Physiol Rev. 2018;98(1):505-53.

23. Sama IE, Ravera A, Santema BT, van Goor H, Ter Maaten JM, Cleland JGF, et al. Circulating plasma concentrations of angiotensin-converting enzyme 2 in men and women with heart failure and effects of renin-angiotensin-aldosterone inhibitors. Eur Heart J. 2020;41:1810-7.

24. Chen X, Cao R, Zhong W. Host calcium channels and pumps in viral infections. Cells. 2019;9(1):94.

25. Fujioka Y, Nishide S, Ose T, Suzuki T, Kato I, Fukuhara H, et al. A sialylated voltage-dependent $\mathrm{Ca}^{2+}$ channel binds hemagglutinin and mediates influenza A virus entry into mammalian cells. Cell Host Microbe. 2018;23(6):809-18.

26. Wasserstrom JA, Kapur S, Jones S, Faruque T, Sharma R, Kelly JE, et al. Characteristics of intracellular $\mathrm{Ca}^{2+}$ cycling in intact rat heart: a comparison of sex differences. Am J Physiol Heart Circ Physiol. 2008;295(5):H1895-904.

27. Machuki JO, Zhang HY, Geng J, Fu L, Adzika GK, Wu L, et al. Estrogen regulation of cardiac cAMP-L-type $\mathrm{Ca}^{2+}$ channel pathway modulates sex differences in basal contraction and responses to $\beta_{2}$ AR-mediated stress in left ventricular apical myocytes. Cell Commun Signal. 2019;17(1):34.

28. Viveiros A, Rasmuson J, Vu J, Mulvagh SL, Yip CYY, Norris CM, Oudit GY. Sex differences in COVID-19: candidate pathways, genetics of ACE2, and sex hormones. Am J Physiol Heart Circ Physiol. 2021;320(1):H296-304.

29. Regitz-Zagrosek V, Kararigas G. Mechanistic pathways of sex differences in cardiovascular disease. Physiol Rev. 2017;97(1):1-37.

30. Deng G, Yin M, Chen X, Zeng F. Clinical determinants for fatality of 44,672 patients with COVID-19. Crit Care. 2020;24(1):179.

31. De Rosa S, Spaccarotella C, Basso C, Calabrò MP, Curcio A, Filardi $\mathrm{PP}$, et al. Reduction of hospitalizations for myocardial infarction in Italy in the COVID-19 era. Eur Heart J. 2020;41(22):2083-8.

32. Du Y, Tu L, Zhu P, Mu M, Wang R, Yang P, et al. Clinical features of 85 fatal cases of COVID-19 from Wuhan. A retrospective observational study. Am J Respir Crit Care Med. 2020;201(11):1372-9.

33. Kim J, Kim DW, Kim KI, Kim HB, Kim JH, Lee YG, et al. Compliance of antihypertensive medication and risk of Coronavirus Disease 2019: a cohort study using big data from the Korean National Health Insurance Service. J Korean Med Sci. 2020;35(25):e232.

34. Yan H, Valdes AM, Vijay A, Wang S, Liang L, Yang S, et al. Role of drugs used for chronic disease management on susceptibility and severity of COVID-19: a large case-control study. Clin Pharmacol Ther. 2020;108:1185-94.

35. Clerkin KJ, Fried JA, Raikhelkar J, Sayer G, Griffin JM, Masoumi A, et al. COVID-19 and cardiovascular disease. Circulation. 2020;141(20):1648-55.

36. Nicin L, Abplanalp WT, Mellentin H. Cell type-specific expression of the putative SARS-CoV-2 receptor ACE2 in human hearts. Eur Heart J. 2020;41(19):1804-6.

37. Lin SY, Lin CL, Lin CC, Hsu WH, Lin CD, Wang IK, et al. Association between Angiotensin-converting enzyme inhibitors and lung cancer-a Nationwide, population-based, propensity score-matched cohort study. Cancers (Basel). 2020;12(3):747.

38. Mechanick JI, Rosenson RS, Pinney SP, Mancini DM, Narula J, Fuster V. Coronavirus and cardiometabolic syndrome: JACC focus seminar. J Am Coll Cardiol. 2020;76(17):2024-35.

39. Little P. Non-steroidal anti-inflammatory drugs and COVID-19. BMJ. 2020;368:m1185.

40. Mancia G, Rea F, Ludergnani M, Apolone G, Corrao G. ReninAngiotensin-Aldosterone system blockers and the risk of Covid-19. N Engl J Med. 2020;382(25):2431-40.

41. Iaccarino G, Grassi G, Borghi C, Ferri C, Salvetti M, Volpe M, et al. Age and multimorbidity predict death among COVID-19 patients: results of the SARS-RAS study of the Italian Society of hypertension. Hypertension. 2020;76(2):366-72. 